\title{
DIFICULTADES DE APRENDIZAJE E INTINERARIO VITAL EN NIÑOS ADOPTADOS: INTERACCIONES E INFLUENCIAS DESDE LA TRIADA M.A.N. (MALTRATO, ABUSOS, NEGLIGENCIA)
}

\author{
Eduardo Barca Enríquez \\ Universidade da Coruña \\ e.barcae@udc.es \\ Alfonso Barca Lozano \\ Universidade da Coruña \\ barca@udc.es
}

\section{RESUMEN}

Los procesos de adopción son mecanismos de protección que la infancia necesita cuando especialmente se aprecian situaciones de maltrato, abuso, negligencia (tríada M.A.N.) por lo cual a los niños adoptados se les pretende facilitar unos contextos de seguridad y cuidados además de proporcionarles la afectividad necesaria, una educación y socialización óptimas, contextos imprescindibles para un desarrollo equilibrado y normalizado para estos niños. Con este trabajo se pretende, primeramente conocer los posibles problemas de aprendizaje y dificultades escolares como consecuencia de un itinerario vital dañino para los niños adoptados con 209 sujetos participantes con características familiares, de desarrollo en sus primeros años de vida y unos contextos adversos con incidencia negativa en su proceso evolutivo vital; después, se analizan las interacciones y relaciones existentes entre variables del itinerario vital y contextos de los primeros meses y primeros años de desarrollo. Finalmente, se busca si hay influencia de ambos tipos de variables sobre la triada MAN y el rendimiento académico. Los resultados muestran la existencia clara de diversas dificultades de aprendizaje en los sujetos participantes en áreas de lectura/escritura, lenguaje oral, de cálculo así como en relación con las interacciones entre las estancias de los primeros años en instituciones y, especialmente, en interacción con la familia biológica y con la familia de acogida/adoptiva. Además, existen correlaciones significativas entre las estancias de los niños en los primeros años y dificultades de aprendizaje en lectura, comprensión y expresión orales. Finalmente, variables como dificultades de cálculo, lugar y estancia del niño en el cuarto año y el primer año de nacimiento poseen relevante influencia tanto sobre la tríada MAN como las dificultades de comprensión y lugar de convivencia de los hijos en el cuarto año sobre el rendimiento académico.

Palabras clave: dificultades de aprendizaje; maltrato; abuso; negligencia (MAN); familia biológica; familia adoptiva; rendimiento académico 


\title{
DIFICULTADES DE APRENDIZAJE E INTINERARIO VITAL EN NIÑOS ADOPTADOS: INTERACCIONES E INFLUENCIAS DESDE LA TRIADA M.A.N. (MALTRATO, ABUSOS, NEGLIGENCIA)
}

\begin{abstract}
Learning disabilities and vital itinerary in children adopted: Interactions and influences from the triad M.A.N. (maltreatment, abuse, negligence). Adoption processes are protection mechanisms that children need when, in particular, situations of maltreatment, abuse, negligence (MAN triad) are observed, whereby adopted children are expected to provide safety and care contexts as well as to provide them with the necessary affectivity, optimal education and socialization, essential contexts for a balanced and normalized development for these children. This paper aims, firstly, to know the possible problems of learning and school difficulties as a consequence of a harmful life itinerary for adopted children with 209 participating subjects with family characteristics, development in their first years of life and adverse contexts with negative impact in its vital evolutionary process; then, the interactions and existing relationships between variables of the vital itinerary and contexts of the first months and first years of development are analyzed. Finally, we look for the influence of both types of variables on the MAN triad and academic achievement. The results show the clear existence of diverse learning difficulties in the subjects participating in reading/writing, oral language, and calculation areas, as well as in relation to the interactions between the first years in institutions and, specially, in interaction with the biological family and the foster/adoptive family. Moreover, there are significant correlations between the stays of children in the first years and learning difficulties in reading, comprehension and oral expression. Finally, variables such as difficulties of calculation, place and stay of the child in the fourth year and the first year of birth have relevant influence on both the MAN triad and the difficulties of understanding and place of coexistence of the children in the fourth year on the academic achievement.
\end{abstract}

Keywords: abuse; maltreatment; negligence (MAN); adoptive family; academic achievement; biological family; learning difficulties

\section{ANTECEDENTES}

En la actualidad se sabe que son muchos los factores que intervienen en el desarrollo cognitivo de un niño aunque éstos pueden estar en exceso representados en los menores adoptados. Sin embargo, la información sobre las condiciones y elementos del contexto próximo en el periodo preadoptivo del niño, con frecuencia es escasa 0 incierta (Dalen, 2011). Las dificultades de aprendizaje 0 trastornos de conducta, tanto en casa como en la escuela, son consecuencia de alteraciones emocionales y vinculares de etapas tempranas que han vivido los menores adoptados por la falta de estimulación psicoafectiva y de apego en las familias de origen 0 en los centros 0 instituciones de protección (Barca, 2015; Mouronte, 2018; Pérez Pichel, 2016).

Una de las causas de lo anterior puede estar referido a que esas dificultades de aprendizaje son habitualmente atribuidas desde la escuela a factores de tipo motivacional y actitudinal, negándoles la oportunidad de disponer de unas pautas educativas distintas al obviar que su aptitud para algunos aprendizajes 0 en algunos procesos cognitivos pueda estar jugando un papel importante. Lo que más preocupa de todo esto es que suelen ser explicaciones apriorísticas, es decir, sin tener ningún tipo de valoración psicopedagógica previa o en el peor de los casos, aun teniéndola y tratando de explicar cómo las dificultades que presenta el chico tienen una base objetiva, se les sigue negando en algunos casos las medidas educativas que realmente necesita, bien por falta de formación o personal habilitado para tal efecto, o bien por falta de voluntad para hacer las cosas de otras maneras, 0 explorar nuevas formas de aprendizaje que sean más útiles para sus alumnos (Barca, 2015; Oropesa, 2017).

Las dos primeras suelen ser las hipótesis explicativas sobre los bajos resultados en estos chicos que participan en el estudio, atendiendo siempre a aspectos de desgana, pobre/mala actitud, falta de interés, distracción, inquietud excesiva, a no querer trabajar, a la falta de esfuerzo, es un 
maleducado, a que las grandes dificultades que tiene, a que le falta base, a que solo aprende lo que le interesa, a la falta de hábito, a la falta de autonomía, en resumen, son muchas las explicaciones según el profesorado pero muy pocas atienden a las dificultades reales que presentan a la hora de procesar la información.

\section{OBJETIVOS}

Se pretende identificar, por una parte, los diferentes problemas de aprendizaje y dificultades escolares característicos de los niños adoptados dentro de la muestra utilizada para poder conocer sus principales necesidades en el contexto escolar y, por otra parte, analizar la posible relación que estos problemas pueden tener con las vivencias tempranas relacionadas con la tríada M.A.N. en su itinerario vital antes de la adopción para ofrecer una atención educativa individualizada y ajustada a las necesidades presentadas.

\section{MÉTODO}

\section{Participantes}

Las familias participantes aportan un total de 209 alumnos con una media de edad de 3.1 años en el momento de la adopción y con una convivencia media con la familia adoptiva de 7.8 años. La proporción entre varones y mujeres es del $56 \%$ el primer caso y $44 \%$ el segundo siendo la gran mayoría de niños provenientes de adopción internacional en un 80\%. Las familias adoptivas y acogedoras (un 10\% del total) provienen de 17 comunidades autónomas de España. Las comunidades autónomas que mayor número de familias adoptivas aportan son las de Andalucía, Cataluña, Madrid, Comunidad Valenciana y Galicia (Ver tabla 1).

En cuanto al tipo de familias con la que residen los sujetos participantes de la investigación predominan las familias adoptivas (92.8\%) sobre las familias de acogida (7.2\%), de manera que de cada diez eran familias adoptivas y solo un $10 \%$ eran familias acogedoras, donde el $80 \%$ de los sujetos eran de adopción internacional y el 20\% de adopción nacional.

Tabla 1. Comunidades autónomas de España en las que residen las familias adoptivas de los sujetos participantes en la investigación (en frecuencias y porcentajes).

\begin{tabular}{|c|c|c|c|c|c|}
\hline & $\mathrm{N}$ & $\%$ & \multicolumn{3}{|c|}{$\%$ acumulado } \\
\hline Andalucía & 45 & 21.5 & & 21.5 & \\
\hline Aragón & & 9 & 4.3 & & 25.8 \\
\hline Asturias & 4 & 1.9 & & 27.7 & \\
\hline Baleares & 1 & 0.5 & & 28.2 & \\
\hline Castilla y León & & 9 & 4.3 & & 32.5 \\
\hline Castilla La Mancha & 12 & 5.7 & & 38.3 & \\
\hline Comunidad Valenciana & & 21 & 10 & & 48.3 \\
\hline Canarias & 4 & 1.9 & & 50.2 & \\
\hline Cantabria & 3 & 1.4 & & 51.6 & \\
\hline Cataluña & 32 & 15.3 & & 66.9 & \\
\hline Extremadura & 13 & 6.2 & & 73.1 & \\
\hline Galicia & 15 & 7.2 & & 80.3 & \\
\hline Madrid & 30 & 14.4 & & 94.7 & \\
\hline Murcia & 2 & 1 & & 95.7 & \\
\hline Navarra & & 4 & 1.9 & & 97.6 \\
\hline País Vasco & 2 & 1 & & 98.6 & \\
\hline La Rioja & 3 & 2.4 & & 100 & \\
\hline
\end{tabular}




\section{Procedimiento e instrumento}

Se ha elaborado la Escala, con denominación Adoescu derivada de la Escala CIVIDA (Barca, 2015), a través de la que se les preguntaba a las familias por el itinerario vital de sus hijos con cuestiones que hacían referencia a temas anteriores y actuales de la adopción y su relación entre estas vivencias y los problemas conductuales que presentaban tanto en el contexto familiar como en el escolar. Se han concretado cuatro áreas de evaluación claras para la obtención de datos relacionados con el estilo e historia de vida de niños adoptados como son, por una parte, los datos relativos a la identificación e interacción de convivencia entre los hijos adoptivos y sus padres; después están aquellos datos sensibles que valoran los posibles malos tratos, abusos y/o negligencias; también se evalúan posibles problemas académicos en casa y en la escuela junto a los rendimientos escolares y posibles dificultades de aprendizaje y, finalmente, un área importante de la existencia de probables trastornos de conducta. Se aplica la Escala tomando los datos de modo online con rigor y solicitando total veracidad por parte de las familias adoptivas y de profesores (ver tablas 2, 3, 4).

\section{Técnicas de análisis}

Se utilizan el paquete estadístico SPSS versión 25 utilizando medidas de tendencia central y análisis correlacionales.

\section{RESULTADOS}

Los resultados se dividen entre la historia previa en los lugares de convivencia de los sujetos en los primeros años y las posibles dificultades y problemas más importantes por los que han pasado. Respecto a los lugares de convivencia en los primeros años en la historia previa de los menores de la muestra, los resultados obtenidos se centrran en que el $48 \%$ había sido separado de su madre biológica en el nacimiento y el resto y hasta los 12 meses la mayoría había convivido en uan institución y con la familia biológica, pasando una mínima parte entre familias de acogida y familias adoptivas. Por tanto, durante el primer años de vida, como dato relevante destacar que entre un $45 \%$ convive en instituiciones y un $32 \%$ con familia biológica, mientras 18\% lo hace con familias de acogida y adoptivas (ver tabla 2). No es despreciable el dato de nacimientos en el hospital así como los datos de convivencia hasta los 12 meses (16.7\% y 9.3\%, respectivamente)

Tabla 2. Frecuencias y porcentajes de sujetos de la muestra en variables relativas al lugar y estancias (lugar nacimiento, lugar en 0-6 meses, 6-12 meses, estancias en el primer año, $2^{\circ}$ año, $3^{\circ}$ año, 4o año) en función del tipo de instituciones y personas de convivencia durante los primeros años de los niños adoptados participantes en la investigación.

\begin{tabular}{|c|c|c|}
\hline Lugar momento & $\mathrm{Ha}$ & Ha vivido \\
\hline Nacimiento & $0-6$ meses & 6-12 meses \\
\hline$\%$ & $\%$ & $\%$ \\
\hline
\end{tabular}

\begin{tabular}{|c|c|c|c|c|c|c|}
\hline Una institución & & 60 & 28.7 & 95 & 45.5 & 102 \\
\hline Familia biológica & 81 & 38.8 & 65 & 31.5 & 55 & 26.3 \\
\hline Familia de acogida & 1 & 0.5 & 7 & 3.3 & 11 & 5.3 \\
\hline Hospital & 35 & 16.7 & 19 & 9.1 & 7 & 3.3 \\
\hline En la calle & 1 & 0.5 & 1 & 0.5 & 1 & 0.5 \\
\hline Se desconoce & 31 & 14.8 & 13 & 6.2 & 13 & 6.2 \\
\hline Familia adoptiva & -- & -- & 9 & 3.9 & 20 & 9.6 \\
\hline Total & 209 & 100 & 209 & 100 & 209 & 100 \\
\hline
\end{tabular}


Si se tiene en cuenta la convivencia de los adoptados a partir del primer, segundo y tercer años se aprecia que permanece todavía alta la convivencia en una 0 alguna institución ( $1^{\circ}$ año: $44.5 \%, 2^{\circ}$ : $33.5 \%$ y $3^{\circ}$ año $23 \%$, respectivamente) y se aprecia que con la familia biológica va disminuyendo (1ำ año: $21 \%, 2^{\circ}$ año: $17 \%$ y $3^{\circ}$ año: $10 \%$, respectivamente), mientras que la convivencia con familias de acogida no deja de ser testimonial $(6.2 \%, 4.8 \%$ y $4.8 \%$ de los adoptados, respectivamente en los tres años). Como contrapartida se aprecia que se incrementa la convivencia con familias adoptivas a medida que van pasando los tres primeros años en porcentajes de $23.9 \%$ y, sobre todo, en el tercer año con un $58.9 \%$ de los menores de la muestra. Si se observa el 4ํ año de la convivencia (ver tabla 2), se aprecia claramente la inversión de los datos anteriores: tan solo el $15.8 \%$ convive en una institución, el 5.2\% con la familia biológica, el 6.7\% con familia de acogida y, lo más importante es que el $71.3 \%$ lo hace ya con familias adoptivas.

Tabla 3. Frecuencias y porcentajes de la muestra en variables relativas al lugar y estancias (lugar de convivencia y estancias en el primer año, 2ำ año, $3^{\circ}$ año, 4a año) en función del tipo de instituciones y personas de convivencia durante los 4 primeros años de los adoptados participantes en la investigación.

$\begin{array}{llll}\text { Ha vivido } & \begin{array}{l}\text { Ha vivido } \\ \text { el } 1^{\circ} \text { año }\end{array} & \text { el } 2^{\circ} \text { año vivido } & \begin{array}{l}\text { Ha vivido } \\ \text { el } 3^{\circ} \text { año }\end{array} \\ \text { el } 4^{\circ} \text { año }\end{array}$

\begin{tabular}{llllllllll}
\hline $\mathrm{N}$ & $\%$ & $\mathrm{~N}$ & $\%$ & $\mathrm{~N}$ & $\%$ & $\mathrm{~N}$ & $\%$ \\
\hline
\end{tabular}

\begin{tabular}{|c|c|c|c|c|c|c|c|c|}
\hline $\begin{array}{l}\text { Una institución } \\
15.8\end{array}$ & & 93 & 44.5 & 70 & 33.5 & 50 & 23.9 & 33 \\
\hline Familia biológica & 44 & 21.1 & 36 & 17.2 & 21 & 10 & 11 & 5.2 \\
\hline Familia de acogida & 13 & 6.2 & 10 & 4.8 & 10 & 4.8 & 14 & 6.7 \\
\hline Hospital & 1 & 0.5 & 1 & 0.5 & -- & -- & 1 & 0.5 \\
\hline En la calle & 1 & 0.5 & 1 & 0.5 & -- & -- & -- & -- \\
\hline Se desconoce & 8 & 3.3 & 6 & 2.9 & 5 & 2.4 & 1 & 0.5 \\
\hline Familia adoptiva & 50 & 23.9 & 1 & 0.5 & 123 & 58.9 & 149 & 71.3 \\
\hline Total & 209 & 100 & 209 & 100 & 209 & 100 & 209 & 100 \\
\hline
\end{tabular}

Pasando al análisis de los datos que reflejan las situaciones del itinerario vital (situaciones de maltrato, abuso y negligencia) asi como las posibles dificultades de aprendizaje que presentan los menores de la muestra, se aprecian datos especialmente relevantes a tener en cuenta. 
Tabla 4. Frecuencias (sí, sí presenta; no, no presenta), porcentajes (\%), Medias, Desviaciones típicas de variables integradas en MAN (maltrato, abuso, negligencia) y variables de dificultades de aprendizaje (lectura, escritura, ortografía, comprensión oral, expresión, cálculo) de los niños adoptados participantes en la investigación.

\begin{tabular}{|c|c|c|c|c|c|c|}
\hline & Sí & $\%$ & No & $\%$ & \multicolumn{2}{|c|}{ Media DT } \\
\hline Ha sufrido maltrato & 44 & 21.3 & 165 & 78.9 & 1.79 & .409 \\
\hline Ha sufrido abusos & 8 & 3.8 & 201 & 3.8 & 1.96 & .192 \\
\hline $\begin{array}{l}\text { Ha sufrido negligencia } \\
.494\end{array}$ & & 122 & 58.3 & 87 & 41.6 & 1.42 \\
\hline MAN (maltrato+abuso+negligencia) & 129 & 61.7 & 80 & 38.2 & 1.47 & .266 \\
\hline $\begin{array}{l}\text { Presenta dificultades en } \\
\text { lectura }\end{array}$ & 75 & 35.8 & 134 & 35.8 & 1.64 & .481 \\
\hline $\begin{array}{l}\text { Presenta dificultades en } \\
\text { escritura }\end{array}$ & 73 & 34.9 & 136 & 65,1 & 1.65 & .478 \\
\hline $\begin{array}{l}\text { Presenta dificultades en } \\
\text { ortografía }\end{array}$ & 58 & 27.7 & 151 & 27.7 & .172 & .449 \\
\hline $\begin{array}{l}\text { Presenta dificultades en } \\
\text { comprensión }\end{array}$ & 88 & 42.1 & 121 & 42.1 & 1.58 & .495 \\
\hline $\begin{array}{l}\text { Presenta dificultades en } \\
\text { expresión }\end{array}$ & 83 & 39.7 & 126 & 39.9 & 1.60 & .490 \\
\hline $\begin{array}{l}\text { Presenta dificultades en } \\
\text { cálculo }\end{array}$ & 81 & 38.7 & 128 & 61.2 & 1.61 & .488 \\
\hline
\end{tabular}

Así, en torno a 6 menores de cada 10 ha sufrido negligencia temprana antes de ser adoptados. El $21 \%$ refiere haber sufrido abusos físicos y un $4,6 \%$ abusos sexuales. Si se observa la tabla 4 se aprecia que sumando negligencia, abusos y malos tratos tenemos las siglas MAN y ello supone que de los 209 sujetos, un total de 129 ha sufrido algún tipo negligencia, abuso o maltrato ("MAN"), Io que supone en el total de la muestra, el porcentaje de $61.7 \%$ de chicos que han sufrido algún maltrato, negligencia o abuso. A nivel académico, solamente uno de cada tres chicos no presentaban dificultades de aprendizaje relevantes, aunque las dificultafdes más comunes fueron problemas de comprensión de lenguaje (42\%), de expresión (39\%) y de cálculo (39\%). Además, uno de cada tres chicos presentaba problemas de lectura y de escritura (35.8\% y $34.9 \%$, respectivamente).

Análisis correlacionales: lugar de residencia y convivencia en relación con maltrato, abusos, negligencia (MAN)

Interesa conocer ahora los coeficientes de correlación, con su posible significatividad, entre los lugares de convivencia en los doce primeros meses y los cuatro primeros años de los menosres de la muestra y en relación con el síndrome MAN (Maltrato, Abuso, Negligencia). Parece interesante este análisis puesto que proporciona una información adicional en este trabajo para la mejor comprensión de la convivencia de los sujetos en relación con su itinerario vital en los cinco primeros años. 
Tabla 4. Coeficientes de correlación entre lugar de nacimiento y estancias de convivencia (lugar nacimiento (Nac.), lugar/estancia en 0-6 meses, 6-12 meses, estancias/convivencia en el primer año, 2ํaño, 3ำ año, $4^{\circ}$ año) y procesos MAN (maltrato, abusos, negligencia) de los niños adoptados participantes en la investigación.

\begin{tabular}{llllllll}
\hline Nac. & $0-6$ & $6-12$ & $\begin{array}{l}1^{\circ} \\
\text { año }\end{array}$ & $\begin{array}{l}2^{\text {o }} \\
\text { año }\end{array}$ & $\begin{array}{l}3^{\circ} \\
\text { año }\end{array}$ & $\begin{array}{l}4^{\circ} \\
\text { año }\end{array}$ & MAN $^{1)}$ \\
\hline
\end{tabular}

Nac. 1

$0-6 \quad 3.92 * * 1$

6-12 $2.13^{* *} .478^{* *} 1$

$1^{\circ} \mathrm{a} \quad 0.99 \quad .164 * \quad .549 * * 1$

$2^{\circ}$ a $\quad .186^{* *} .118 \quad .383^{* *} .657^{* *} 1$

$3^{\mathrm{o}}$ a $\quad 0.91 * * 0.13 \quad .186^{* *} .385^{* *} .640 * * 1$

$\begin{array}{lllll}4^{\mathrm{o}} \mathrm{a} & 0.73 & -.017 & .078 & .225 * * .417 * * .707 * * 1\end{array}$

$\begin{array}{llll}\text { MAN }^{1)} 0.70 & -.0 .10 & .082 & .193 * * .223^{* *} .211^{* *} .264 * * 1\end{array}$

$\mathrm{MAN}^{1)}$ : Maltrato, abusos, negligencia

${ }^{*} p<.05$ (bilateral); ${ }^{* *} p<.001$ (bilateral)

En cuanto al lugar de nacimiento y estancia/convivencia en los seis primeros meses (0-6) y hasta los 12 meses, en su correlación con Maltrato, Abusos, Negligencia (MAN) se aprecia en la tabla 4 cómo existe una correlación positiva aunque muy baja ye incluso negativa, pero sin trascen dencia alguna. Sin embargo, en los cuatro años siguientes los coeficientes de correlación de las estancias/convivencias se incrementan y todos ellos son significativos $(p<.000)$ en relación con las situaciones de maltrato, abuso y negligencia. Todo ello significa que estas "situaciones MAN" en niños adoptados parece que tienen lugar con mayor intensidad durante los cuatro primeros años de vida de los menores dado que los ceficientes de correlación se van incrementado con los años (ver tabla 4). En consecuencia, cabe pensar que son estas edades las más proclives a sufrir conductas dañinas y experiencias traumáticas tempranas para los menores adoptados.

Análisis correlacionales: dificultades de aprendizaje en relación con Maltrato, Abusos, Negligencia (MAN) 
Tabla 5. Coeficientes de correlación entre dificultades de aprendizaje (lectura, escritura, ortografía, comprensión oral, expresión oral, cálculo) y procesos MAN (maltrato, abusos, negligencia) en los participantes de la investigación.

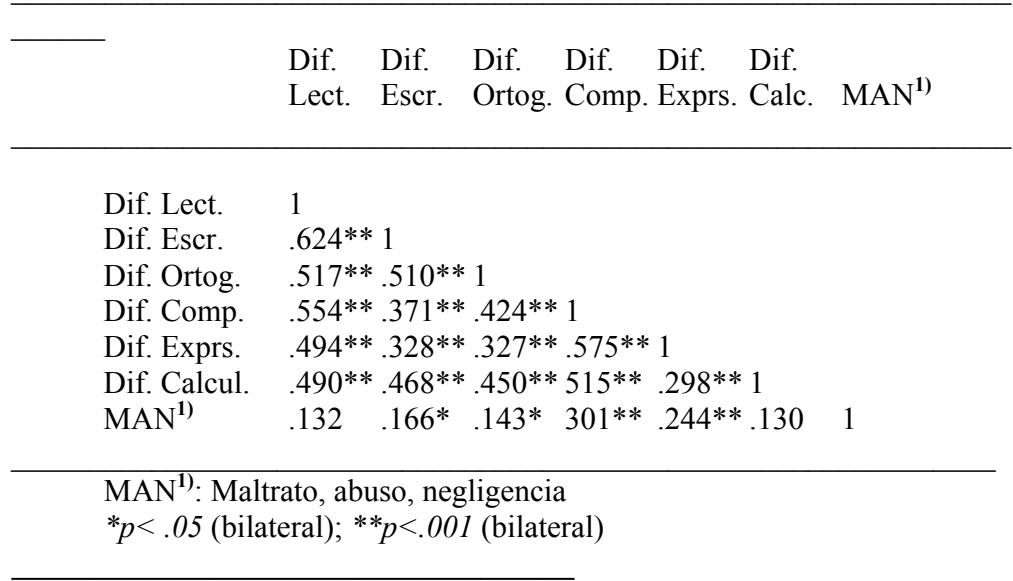

A nivel de análisis correlacionales entre las principales dificultades de aprendizazje encontradas en menores adoptados y situaciones de maltrato, abusos, negligencia (MAN) destacan, en primer lugar los altos coeficientes de correlación existentes entre ellas, especialmente las dificultades en lectura y escritura con las dificultades en comprensión y expresión del lenguaje, pero también es importante apuntar los niveles de correlación siginificativos de las dificultades de aprendizaje de la escritutra, ortografía, comprensión y expresión del lenguaje con las experiencias dañinas de maltrato, abuso y negligencia (situaciones "MAN") de los menores adoptados que integran esta investigación, con lo cual cabe pensar que está contrastado empíricamente que dichas experiencias mantienen afinidades y una relación estrecha con la aparición y mantenimiento de dificultades de aprendizaje centradas en torno a las áreas del lenguaje oral y escrito (ver tabla 5).

\section{DISCUSIÓN Y CONCLUSIONES}

En resumen, se comprueba que están presentes en los menores adoptados tres tipos de dificultades de aprendizaje intimamente relacionadas; por una parte las que se encuentran ligadas a sus lugares de residencia/convivencia, con frecuencia desfavorables para el desarrollo, durante los primeros meses y años; por otra parte se sabe que las dificultades de aprendizaje predominantes son las dificultades de lectura y escritura así como las de comprensión y expresión del lenguaje y de cálculo y, finalmente, las tres situaciones dañinas de maltrato, abuso y negligencia están con mucha freceuncia presentes a la hora de describir y explicar dichas dificultades de aprendizaje. Sin embargo, el investigador no debería quedarse solamente con el señalamiento de los déficits funcionales de las dificultades encontradas, sino que debería tratar de ir más allá e incidir en los procesos cognitivos, afectivos y motivacionales que están detrás de estos déficits y que afectan al conjunto de la personalidad de los menores (Barca, 2015; Barca, Brenlla y Ramudo, 2017) .

Otros autores señalan que existen también posibles factores de riesgo que explican ciertas causas como responsables de las dificultades de aprendizaje en niños adoptados, entre ellas están la exposición a drogas y al alcohol antes del nacimiento, complicaciones en el momento del nacimiento y la nutrición deficiente, además de la negligencia o abusos (Morin, 2019). Del mismo modo se llega 
conclusiones semejantes cuando se señala que hay un elevado número de dificultades de aprendizaje en niños adoptados que se relacionan con dos hechos relevantes como son la alta posibilidad de haber estado viviendo en una institución en edades tempranas así como con el hecho de haber sufrido vivencias traumáticas como maltrato, abusos físicos/psicológicos, abusos sexuales y negligencia (Mouronte, 2018: pp. 21-22).

Por su parte Janire Rojas (2015) considera que la vida de un niño antes de la adopción puede estar afectada en el campo personal, académico y social, lo que puede provocar a medio y largo plazo autoestima baja, falta de autocontrol y frustracion.

Como recursos educativos para procesos de intervención y ayuda a menores adoptados hay medios como manuales específicos que ayudan a trabajar con una metodología eficaz en el aula con menores (Geddes, 2010; Gonzalo, 2012), así como otras orientaciones para maestros y profesores que ya han demostrado buenos resultados (Múgica, 2010).

Desde hace varios años se ha observado que en el momento en que las intervenciones educativas con niños adoptados se diseñan incluyendo la dimensión afectiva (se prima la confianza entre ambos, la sintonía y la tranquilidad dentro de esa relación) entre el alumno y el profesional, ya sea profesor, psicopedagogo o psicólogo, y la dimensión motivacional centrada en el uso de materiales y contenidos que partan de sus intereses, los resultados obtenidos en cuanto a la calidad y cantidad de aprendizaje por parte del alumno son realmente prometedores y considero que digno de estudios para el futuro y así diseñar terapéuticas escolares y conductuales realmente eficaces y productivas para estos chicos.

Es preciso entender que detrás de esas dificultades de aprendizaje no hay incompetencias parentales ni problemas actitudinales sino unas crianzas de un elevado nivel de exigencia, aunque desde todos los actores se deberá promover actividades de formación educativa especializada para saber cómo trabajar y atender las necesidades del "niño dañado". En realidad se resumen en tres grandes núcleos (Barudy y Dantagnan, 2010; Barca; 2015; Barca et al., 2017), donde, por una parte, hay que lograr la identificación de sus dificultades desde una explicación comprehensiva y coherente con su recorrido vital; por otra parte, buscar el desarrollo de estrategias de relación y vinculación eficaces y cualitativamente distintas de las que se utilizan actualmente para la mayoría de los niños, para su promoción con padres y educadores y, por último, la adaptación de contenidos curriculares y la creación de nuevas didácticas, estrategias de aprendizaje y metodologías ajustadas a sus necesidades reales especialmente en habilidades para la comprensión lectora (Alfonso et al. (2017) que igualmente, distan, en aquellos que presentan dificultades escolares de las que se utilizan para el niño normal y de las dificultades de aprendizaje que se conocen hasta el momento.

\section{REFERENCIAS BIBLIOGRÁFICAS}

Alfonso, S., Deaño, M., Ramos, A., Conde, A.; Gayo, M. E., Iglesias-Sarmiento, Limia, S., \& Tellado, F. (2017). Pevención e intervencion de las dificultades de aprendizaje en lectura. Revista Infad de Psicología. International Journal of Developmental and Educational Psychology, no1, (vol.1) 71-81. DOI: http://dx.doi.org/10.17060/ijodaep.2017.n1,v3.976.

Barca, E. (2014). Evaluación comprehensiva del niño adoptado: resignificando síntomas. En R. Ma․ Fernández García (Coord.). Neuropsicología del abandono y el maltrato infantil. Pp. 55-65. Barcelona: Hilo Rojo Ediciones.

Barca, E. (2015). Análisis del impacto y la alteración de la función cognitiva en niños adoptados y/o víctimas del Maltrato, Abuso y/o Negligencia temprana desde el modelo PASS de procesamiento de la información. Ourense: Universidad de Vigo (Tesis doctoral inédita).

Barca, E., Brenlla, J. C., \& Ramudo, I. (2017). Variables críticas pre-adopción y dificultades de apren- 
dizaje. Revista de Estudios e Investigación en Psicología y Educación, (01), 224-230. https://doi.org/10.17979/reipe.2017.0.01.2606

Barudy, J. \& Dantagnan, M. (2010). Los desafíos invisibles de ser padre o madre: manual de evaluación de las competencias y la resiliencia parental. Barcelona: Gedisa.

Dalen, M. (2011). Desarrollo cognitivo y logro educativo en personas adoptadas internacionalmente. I Fórum Internacional sobre Infancias y Familias. "De filias y fobias". Del parentesco biológico al cultural. Barcelona, 29 y 30 de septiembre y 2 y 3 de octubre de 2006 . Recuperado de: http://www.ciimu.org/webs/foruminternacional/

Geddes, H. (2010). El apego en el aula: revlación entre las primeras experiencias infavntiles, el bienestar emocional y el rednimiento escolar. Barcelona: Graó.

Gonzalo, J.L. (2012). ¿Todo niño viene con un pan debajo del brazo?. Guía para padres adoptivos con hijos con trastornos de apego. Bilbao: Desclée de Brouwer.

Morin, A. (2019). Dificultades de aprendiuzaje y de atención en niños adoptados. Recuperado de https://www.understood.org/es-mx/learning-attention-issues/getting-started/what-you-need-toknow/learning-and-attention-issues-in-adopted-children

Mouronte, L. (2018). Dificultades de aprendizaje escolar en niños adoptados. 26 Pp. Trabajo Fin de Máster en Psicología Aplicada. A Coruña: Facultad de Ciencias de la Educación. Universidade da Coruña.

Múgica, J. (2010), Hacia una escuela inclusiva: el reto de integrar a niños y niñas socialmente desfavorecidos. Irún: Seminario de Berritzegune de Irún. Recuperado de : http://g02.berritzeguneak.net/es/

Oropesa, N.F. (2017). Tipología de familias adoptivas y seguridad en las conductas de apego infantil. Revista de Psicología Infad. International Journal of Developmental and Educational Psychology, vol.1, no 1. 327-339. D0I: http://dx.doi.org/10.17060.ijodaep.2017.n1.v31001

Pérez Pichel, M. (2016). Hacer familia. Adopción: seis problemas frecuentes de los niños adoptados. Recuperado de https://www.hacerfamilia.com/ninos/noticia-adopcion-problemas-frecuentes-ninos-adoptados-20160923143720.htm

Rojas, J. (2015 ). Por qué los niños adoptados tienen dificultades académicas, en AUSARTI, marzo, 3. Recuperado de https://ausarti.com/por-que-los-ninos-adoptados-tienen-dificultades-academicas-2/ 